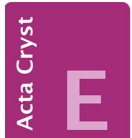
COMMUNICATIONS

ISSN 2056-9890

\section{Crystal structure of 2-methylsulfanyl-1- (thiomorpholin-4-yl)ethanone}

\author{
Gihaeng Kang, Jineun Kim,* Eunjin Kwon and Tae Ho \\ Kim*
}

Department of Chemistry and Research Institute of Natural Sciences, Gyeongsang, National University, Jinju 52828, Republic of Korea. *Correspondence e-mail: thkim@gnu.ac.kr, jekim@gnu.ac.kr

Received 12 August 2015; accepted 18 August 2015

Edited by W. T. A. Harrison, University of Aberdeen, Scotland

In the title compound, $\mathrm{C}_{7} \mathrm{H}_{13} \mathrm{NOS}_{2}$, the thiomorpholine ring adopts a chair conformation and the bond-angle sum at the $\mathrm{N}$ atom is $360^{\circ}$. The dihedral angle between the amide group and the thiomorpholine ring (all atoms) is $36.48(12)^{\circ}$. In the crystal, $\mathrm{C}-\mathrm{H} \cdots \mathrm{O}$ and $\mathrm{C}-\mathrm{H} \cdots \mathrm{S}$ hydrogen bonds link adjacent molecules, forming two-dimensional networks extending parellel to the (011) plane.

Keywords: crystal structure; thiomorpholine; hydrogen bonding.

CCDC reference: 1419333

\section{Related literature}

For further information on the synthesis, see: Kim et al. (2008). For related crystal structures, see: Kim et al. (2006); Ujam et al. (2010).

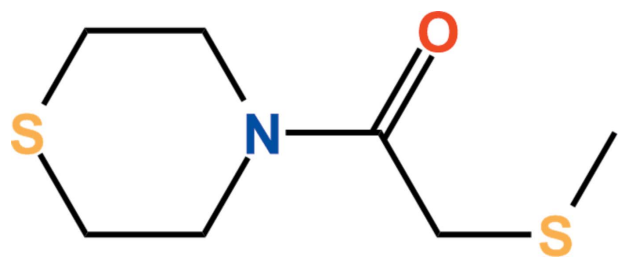

\section{Experimental}

\subsection{Crystal data}

$\mathrm{C}_{7} \mathrm{H}_{13} \mathrm{NOS}_{2}$

$M_{r}=191.30$

Monoclinic, $P 2_{1} / c$

$$
\begin{aligned}
& a=15.0461(15) \AA \\
& b=6.1525(6) \AA \\
& c=10.4751(10) \AA
\end{aligned}
$$

$\beta=107.581(6)^{\circ}$

$V=924.40(16) \AA^{3}$

$Z=4$

Mo $K \alpha$ radiation

$\mu=0.52 \mathrm{~mm}^{-1}$

$T=173 \mathrm{~K}$

$0.23 \times 0.18 \times 0.08 \mathrm{~mm}$

2.2. Data collection

Bruker APEXII CCD diffractometer

Absorption correction: multi-scan (SADABS; Bruker, 2013)

$T_{\min }=0.890, T_{\max }=0.959$

8512 measured reflections 2111 independent reflections 1865 reflections with $I>2 \sigma(I)$ $R_{\text {int }}=0.026$

\subsection{Refinement}

$R\left[F^{2}>2 \sigma\left(F^{2}\right)\right]=0.029$

$w R\left(F^{2}\right)=0.078$

$S=1.05$

2111 reflections
101 parameters

$\mathrm{H}$-atom parameters constrained

$\Delta \rho_{\max }=0.22{\mathrm{e} \AA^{-3}}^{-3}$

$\Delta \rho_{\min }=-0.27$ e $\AA^{-3}$
Table 1

Hydrogen-bond geometry $\left(\AA{ }^{\circ}\right)$.

\begin{tabular}{lllll}
\hline$D-\mathrm{H} \cdots A$ & $D-\mathrm{H}$ & $\mathrm{H} \cdots A$ & $D \cdots A$ & $D-\mathrm{H} \cdots A$ \\
\hline $\mathrm{C} 1-\mathrm{H} 1 B \cdots \mathrm{O} 1^{\mathrm{i}}$ & 0.99 & 2.46 & $3.3490(19)$ & 150 \\
$\mathrm{C} 6-\mathrm{H} 6 B \cdots \mathrm{O} 1^{\mathrm{i}}$ & 0.99 & 2.59 & $3.4427(18)$ & 144 \\
$\mathrm{C} 7-\mathrm{H} 7 B \cdots \mathrm{O} 1^{\mathrm{ii}}$ & 0.98 & 2.45 & $3.3237(19)$ & 148 \\
$\mathrm{C} 3-\mathrm{H} 3 A \cdots \mathrm{S} 2^{\mathrm{iii}}$ & 0.99 & 2.88 & $3.8201(15)$ & 159
\end{tabular}

Symmetry codes: (i) $x,-y+\frac{3}{2}, z+\frac{1}{2}$; (ii) $x, y+1, z$; (iii) $x, y-1, z$.

Data collection: APEX2 (Bruker, 2013); cell refinement: SAINT (Bruker, 2013); data reduction: $S A I N T$; $\operatorname{program}(\mathrm{s})$ used to solve structure: SHELXS97 (Sheldrick, 2008); program(s) used to refine structure: SHELXL2013 (Sheldrick, 2015); molecular graphics: DIAMOND (Brandenburg, 2010); software used to prepare material for publication: SHELXTL (Sheldrick, 2008).

\section{Acknowledgements}

This research was supported by the Basic Science Research Program through the National Research Foundation of Korea (NRF) funded by the Ministry of Education, Science and Technology (No. 2014R1A1A4A01009105).

Supporting information for this paper is available from the IUCr electronic archives (Reference: HB7480).

\section{References}

Brandenburg, K. (2010). DIAMOND. Crystal Impact GbR, Bonn, Germany. Bruker (2013). APEX2, SAINT and SADABS. Bruker AXS Inc., Madison, Wisconsin, USA.

Kim, T. H., Shin, Y. W., Jung, J. H., Kim, J. S. \& Kim, J. (2008). Angew. Chem. Int. Ed. 47, 685-688.

Kim, T. H., Shin, Y. W., Lee, S. S. \& Kim, J. (2006). Anal. Sci. 22, x287-x288. Sheldrick, G. M. (2008). Acta Cryst. A64, 112-122.

Sheldrick, G. M. (2015). Acta Cryst. C71, 3-8.

Ujam, O. T., Devoy, S. M., Henderson, W., Nicholson, B. K. \& Hor, T. S. A. (2010). Inorg. Chim. Acta, 363, 3558-3568. 


\section{supporting information}

Acta Cryst. (2015). E71, o679 [https://doi.org/10.1107/S2056989015015418]

\section{Crystal structure of 2-methylsulfanyl-1-(thiomorpholin-4-yl)ethanone}

\section{Gihaeng Kang, Jineun Kim, Eunjin Kwon and Tae Ho Kim}

\section{S1. Comment}

\section{S2. Experimental}

Thionyl chloride ( $2.38 \mathrm{~g}, 20.0 \mathrm{mmol})$ was added dropwise to 2-methylthioacetic acid ( $2.12 \mathrm{~g}, 20.0 \mathrm{mmol})$ in the pesence of triethylamine ( $2.02 \mathrm{~g}, 20.0 \mathrm{mmol}$ ) in chloroform. The mixture was refluxed for $2 \mathrm{~h}$ and cooled down to room temperature. Then, thiomorpholine $(2.38 \mathrm{~g}, 20.0 \mathrm{mmol})$ and triethylamine $(2.02 \mathrm{~g}, 20.0 \mathrm{mmol})$ in chloroform were added dropwise to the resulting acid chloride solution, cooled by salt and ice water. The solution was stirred for $2 \mathrm{~h}$, and then water was added. Organic layer was collected and water layer was extracted with chloroform. The combined organic layers dried with anhydrous sodium sulfate were evaporated to give crude oil. Column chromatography (silica gel, ethyl acetate/hexane $\left.=20 / 80(v / v), R_{\mathrm{f}} 0.1\right)$ gave pure title compound (3.42 g, 89\%) (Kim et al., 2008). Slow evaporation of a solution in acetone/ethyl acetate gave colourless blocks.

\section{S3. Refinement}

All H-atoms were positioned geometrically and refined using a riding model with $\mathrm{d}(\mathrm{C}-\mathrm{H})=0.99 \AA, U_{\text {iso }}=1.2 U_{\text {eq }}(\mathrm{C})$ for $\mathrm{CH}_{2}$ groups and $\mathrm{d}(\mathrm{C}-\mathrm{H})=0.98 \AA, U_{\text {iso }}=1.5 U_{\mathrm{eq}}(\mathrm{C})$ for $\mathrm{CH}_{3}$ group.

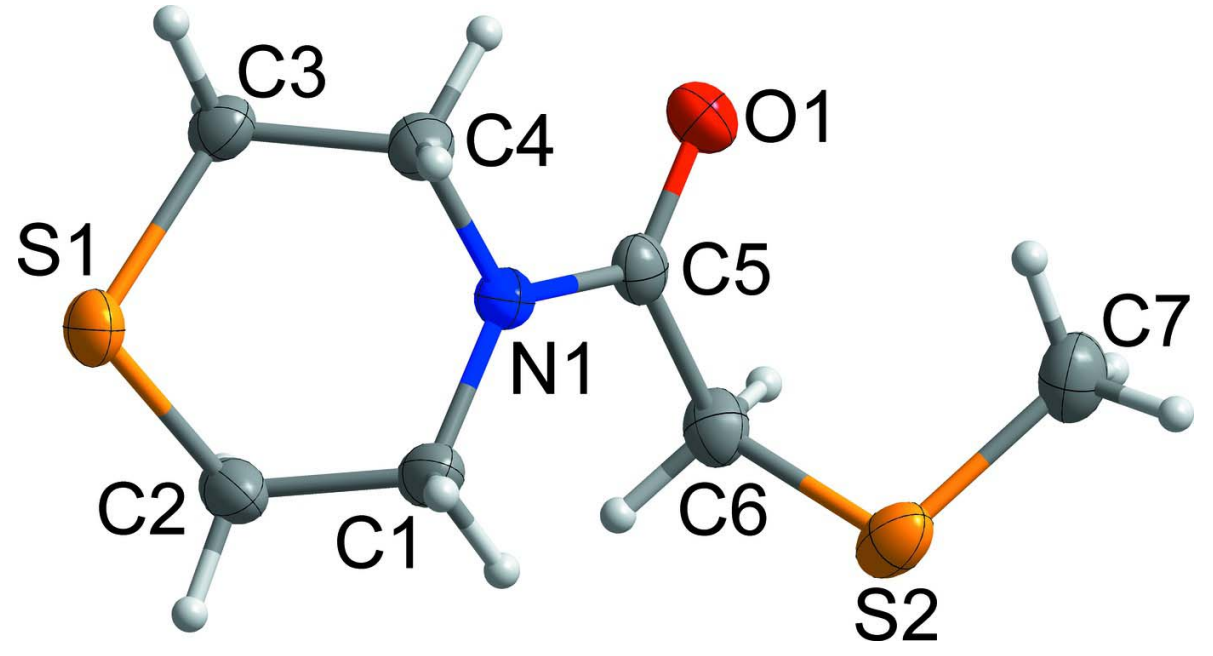

Figure 1

The asymmetric unit of the title compound with displacement ellipsoids drawn at the $50 \%$ probability level. 


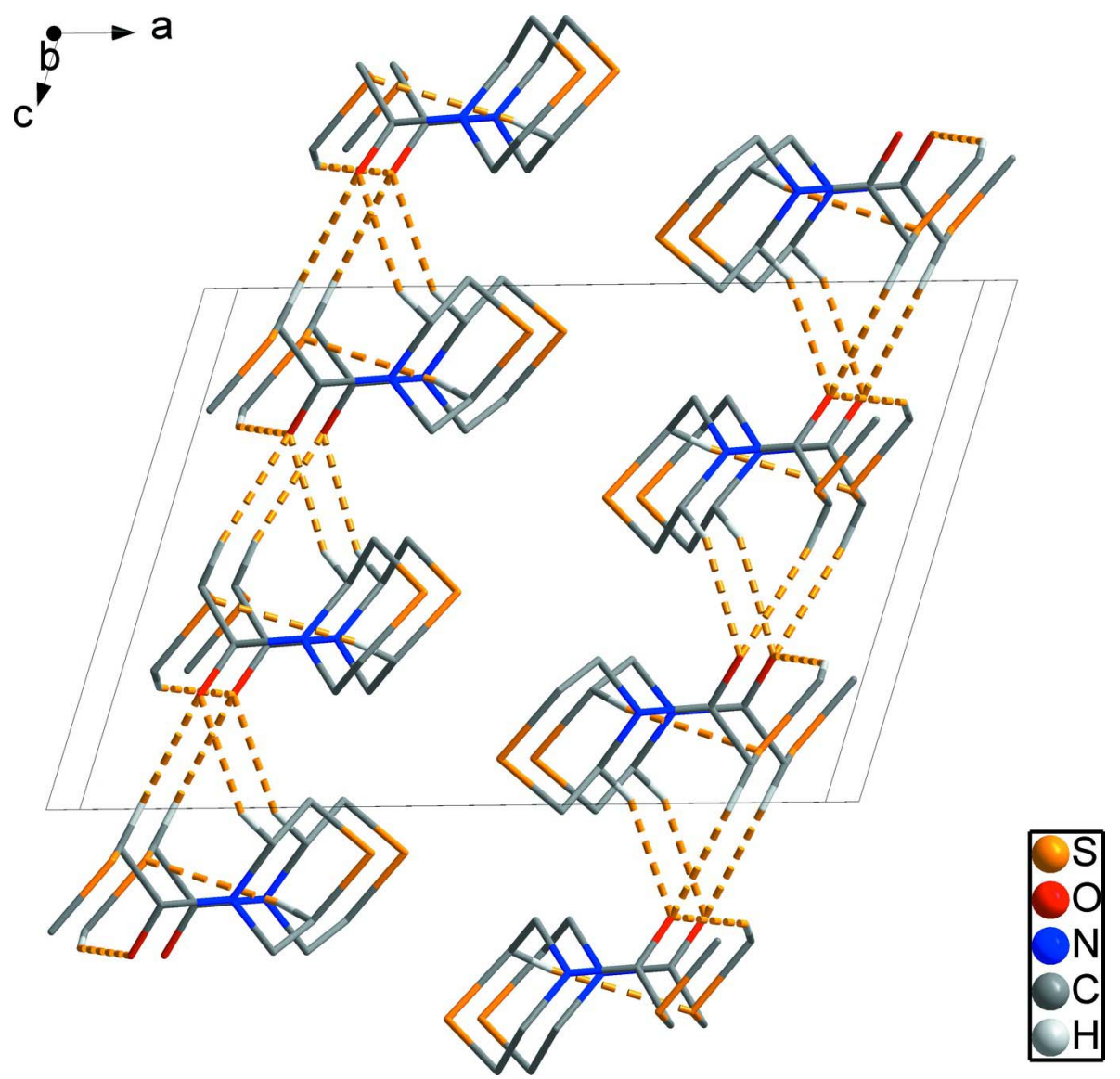

Figure 2

Crystal packing viewed along the $b$ axis. The intermolecular $\mathrm{C}-\mathrm{H} \cdots \mathrm{O}$ and $\mathrm{C}-\mathrm{H} \cdots \mathrm{S}$ hydrogen bonds are shown as dashed lines.

\section{2-Methylsulfanyl-1-(thiomorpholin-4-yl)ethanone}

\section{Crystal data}

\section{$\mathrm{C}_{7} \mathrm{H}_{13} \mathrm{NOS}_{2}$}

$M_{r}=191.30$

Monoclinic, $P 2_{1} / c$

$a=15.0461(15) \AA$

$b=6.1525(6) \AA$

$c=10.4751(10) \AA$

$\beta=107.581(6)^{\circ}$

$V=924.40(16) \AA^{3}$

$Z=4$

\section{Data collection}

Bruker APEXII CCD diffractometer

$\varphi$ and $\omega$ scans

Absorption correction: multi-scan

(SADABS; Bruker, 2013)

$T_{\min }=0.890, T_{\max }=0.959$

8512 measured reflections
$F(000)=408$

$D_{\mathrm{x}}=1.375 \mathrm{Mg} \mathrm{m}^{-3}$

Mo $K \alpha$ radiation, $\lambda=0.71073 \AA$

Cell parameters from 4186 reflections

$\theta=2.8-27.5^{\circ}$

$\mu=0.52 \mathrm{~mm}^{-1}$

$T=173 \mathrm{~K}$

Block, colourless

$0.23 \times 0.18 \times 0.08 \mathrm{~mm}$

2111 independent reflections

1865 reflections with $I>2 \sigma(I)$

$R_{\text {int }}=0.026$

$\theta_{\max }=27.5^{\circ}, \theta_{\min }=2.8^{\circ}$

$h=-19 \rightarrow 19$

$k=-7 \rightarrow 7$

$l=-13 \rightarrow 13$ 


\section{Refinement}

Refinement on $F^{2}$

Least-squares matrix: full

$R\left[F^{2}>2 \sigma\left(F^{2}\right)\right]=0.029$

$w R\left(F^{2}\right)=0.078$

$S=1.05$

2111 reflections

101 parameters

0 restraints
Hydrogen site location: inferred from neighbouring sites

$\mathrm{H}$-atom parameters constrained

$w=1 /\left[\sigma^{2}\left(F_{\mathrm{o}}^{2}\right)+(0.0406 P)^{2}+0.2667 P\right]$

where $P=\left(F_{\mathrm{o}}^{2}+2 F_{\mathrm{c}}{ }^{2}\right) / 3$

$(\Delta / \sigma)_{\max }=0.001$

$\Delta \rho_{\max }=0.22$ e $\AA^{-3}$

$\Delta \rho_{\min }=-0.27$ e $\AA^{-3}$

Special details

Geometry. All e.s.d.'s (except the e.s.d. in the dihedral angle between two l.s. planes) are estimated using the full covariance matrix. The cell e.s.d.'s are taken into account individually in the estimation of e.s.d.'s in distances, angles and torsion angles; correlations between e.s.d.'s in cell parameters are only used when they are defined by crystal symmetry. An approximate (isotropic) treatment of cell e.s.d.'s is used for estimating e.s.d.'s involving 1.s. planes.

Fractional atomic coordinates and isotropic or equivalent isotropic displacement parameters $\left(\AA^{2}\right)$

\begin{tabular}{lllll}
\hline & $x$ & $y$ & $z$ & $U_{\text {iso }} * / U_{\text {eq }}$ \\
\hline S1 & $0.57059(2)$ & $0.35556(6)$ & $0.41078(4)$ & $0.03060(12)$ \\
S2 & $0.87043(3)$ & $1.06954(6)$ & $0.40092(4)$ & $0.03528(13)$ \\
O1 & $0.82963(8)$ & $0.59419(17)$ & $0.21785(11)$ & $0.0333(2)$ \\
N1 & $0.72025(8)$ & $0.62668(19)$ & $0.32412(12)$ & $0.0264(3)$ \\
C1 & $0.68637(10)$ & $0.7095(2)$ & $0.43112(15)$ & $0.0315(3)$ \\
H1A & 0.6300 & 0.7989 & 0.3920 & $0.038^{*}$ \\
H1B & 0.7346 & 0.8037 & 0.4909 & $0.038^{*}$ \\
C2 & $0.66298(11)$ & $0.5254(3)$ & $0.51228(15)$ & $0.0325(3)$ \\
H2A & 0.6437 & 0.5870 & 0.5871 & $0.039^{*}$ \\
H2B & 0.7193 & 0.4357 & 0.5510 & $0.039^{*}$ \\
C3 & $0.62268(10)$ & $0.2963(2)$ & $0.28028(15)$ & $0.0280(3)$ \\
H3A & 0.6779 & 0.2023 & 0.3172 & $0.034^{*}$ \\
H3B & 0.5774 & 0.2148 & 0.2078 & $0.034^{*}$ \\
C4 & $0.65213(10)$ & $0.5000(2)$ & $0.22214(14)$ & $0.0296(3)$ \\
H4A & 0.6795 & 0.4586 & 0.1507 & $0.036^{*}$ \\
H4B & 0.5965 & 0.5909 & 0.1812 & $0.036^{*}$ \\
C5 & $0.80749(9)$ & $0.6585(2)$ & $0.31488(14)$ & $0.0243(3)$ \\
C6 & $0.87815(10)$ & $0.7787(2)$ & $0.42581(15)$ & $0.0297(3)$ \\
H6A & 0.9416 & 0.7294 & 0.4300 & $0.036^{*}$ \\
H6B & 0.8679 & 0.7434 & 0.5125 & $0.036^{*}$ \\
C7 & $0.91981(12)$ & $1.0968(3)$ & $0.26585(18)$ & $0.0385(4)$ \\
H7A & 0.9862 & 1.0593 & 0.2975 & $0.058^{*}$ \\
H7B & 0.9127 & 1.2471 & 0.2334 & $0.058^{*}$ \\
H7C & 0.8876 & 0.9988 & 0.1928 & $0.058^{*}$ \\
& & & & \\
& & & &
\end{tabular}

Atomic displacement parameters $\left(\AA^{2}\right)$

\begin{tabular}{lllllll}
\hline & $U^{11}$ & $U^{22}$ & $U^{33}$ & $U^{12}$ & $U^{13}$ & $U^{23}$ \\
\hline S1 & $0.0255(2)$ & $0.0330(2)$ & $0.0357(2)$ & $-0.00719(13)$ & $0.01283(16)$ & $-0.00026(15)$ \\
S2 & $0.0262(2)$ & $0.0305(2)$ & $0.0515(3)$ & $-0.00455(14)$ & $0.01529(18)$ & $-0.01097(16)$
\end{tabular}




\begin{tabular}{lllllll}
$\mathrm{O} 1$ & $0.0347(6)$ & $0.0363(5)$ & $0.0344(6)$ & $-0.0013(4)$ & $0.0187(5)$ & $-0.0022(4)$ \\
$\mathrm{N} 1$ & $0.0262(6)$ & $0.0290(6)$ & $0.0263(6)$ & $-0.0077(5)$ & $0.0115(5)$ & $-0.0052(5)$ \\
$\mathrm{C} 1$ & $0.0313(8)$ & $0.0309(7)$ & $0.0369(8)$ & $-0.0085(6)$ & $0.0173(7)$ & $-0.0101(6)$ \\
$\mathrm{C} 2$ & $0.0323(8)$ & $0.0403(8)$ & $0.0279(8)$ & $-0.0093(6)$ & $0.0134(6)$ & $-0.0064(6)$ \\
$\mathrm{C} 3$ & $0.0251(7)$ & $0.0268(7)$ & $0.0325(8)$ & $-0.0050(5)$ & $0.0092(6)$ & $-0.0058(6)$ \\
$\mathrm{C} 4$ & $0.0288(7)$ & $0.0341(7)$ & $0.0248(7)$ & $-0.0081(6)$ & $0.0065(6)$ & $-0.0028(6)$ \\
$\mathrm{C} 5$ & $0.0251(7)$ & $0.0212(6)$ & $0.0278(7)$ & $0.0011(5)$ & $0.0099(6)$ & $0.0051(5)$ \\
$\mathrm{C} 6$ & $0.0229(7)$ & $0.0349(7)$ & $0.0300(8)$ & $-0.0034(6)$ & $0.0059(6)$ & $0.0037(6)$ \\
$\mathrm{C} 7$ & $0.0374(9)$ & $0.0309(8)$ & $0.0490(10)$ & $-0.0021(6)$ & $0.0157(8)$ & $0.0058(7)$ \\
\hline
\end{tabular}

Geometric parameters $\left(\AA,{ }^{\circ}\right)$

\begin{tabular}{|c|c|c|c|}
\hline $\mathrm{S} 1-\mathrm{C} 2$ & $1.8061(15)$ & $\mathrm{C} 2-\mathrm{H} 2 \mathrm{~B}$ & 0.9900 \\
\hline $\mathrm{S} 1-\mathrm{C} 3$ & $1.8065(14)$ & $\mathrm{C} 3-\mathrm{C} 4$ & $1.517(2)$ \\
\hline $\mathrm{S} 2-\mathrm{C} 7$ & $1.7935(17)$ & $\mathrm{C} 3-\mathrm{H} 3 \mathrm{~A}$ & 0.9900 \\
\hline $\mathrm{S} 2-\mathrm{C} 6$ & $1.8067(16)$ & $\mathrm{C} 3-\mathrm{H} 3 \mathrm{~B}$ & 0.9900 \\
\hline $\mathrm{O} 1-\mathrm{C} 5$ & $1.2267(17)$ & $\mathrm{C} 4-\mathrm{H} 4 \mathrm{~A}$ & 0.9900 \\
\hline $\mathrm{N} 1-\mathrm{C} 5$ & $1.3592(17)$ & $\mathrm{C} 4-\mathrm{H} 4 \mathrm{~B}$ & 0.9900 \\
\hline $\mathrm{N} 1-\mathrm{C} 1$ & $1.4563(17)$ & $\mathrm{C} 5-\mathrm{C} 6$ & $1.511(2)$ \\
\hline $\mathrm{N} 1-\mathrm{C} 4$ & $1.4622(18)$ & C6-H6A & 0.9900 \\
\hline $\mathrm{C} 1-\mathrm{C} 2$ & $1.520(2)$ & $\mathrm{C} 6-\mathrm{H} 6 \mathrm{~B}$ & 0.9900 \\
\hline $\mathrm{C} 1-\mathrm{H} 1 \mathrm{~A}$ & 0.9900 & $\mathrm{C} 7-\mathrm{H} 7 \mathrm{~A}$ & 0.9800 \\
\hline $\mathrm{C} 1-\mathrm{H} 1 \mathrm{~B}$ & 0.9900 & $\mathrm{C} 7-\mathrm{H} 7 \mathrm{~B}$ & 0.9800 \\
\hline $\mathrm{C} 2-\mathrm{H} 2 \mathrm{~A}$ & 0.9900 & $\mathrm{C} 7-\mathrm{H} 7 \mathrm{C}$ & 0.9800 \\
\hline $\mathrm{C} 2-\mathrm{S} 1-\mathrm{C} 3$ & $97.45(6)$ & $\mathrm{H} 3 \mathrm{~A}-\mathrm{C} 3-\mathrm{H} 3 \mathrm{~B}$ & 107.8 \\
\hline $\mathrm{C} 7-\mathrm{S} 2-\mathrm{C} 6$ & $100.51(7)$ & $\mathrm{N} 1-\mathrm{C} 4-\mathrm{C} 3$ & $111.87(12)$ \\
\hline $\mathrm{C} 5-\mathrm{N} 1-\mathrm{C} 1$ & $125.07(12)$ & $\mathrm{N} 1-\mathrm{C} 4-\mathrm{H} 4 \mathrm{~A}$ & 109.2 \\
\hline $\mathrm{C} 5-\mathrm{N} 1-\mathrm{C} 4$ & $120.24(11)$ & $\mathrm{C} 3-\mathrm{C} 4-\mathrm{H} 4 \mathrm{~A}$ & 109.2 \\
\hline $\mathrm{C} 1-\mathrm{N} 1-\mathrm{C} 4$ & $114.68(11)$ & $\mathrm{N} 1-\mathrm{C} 4-\mathrm{H} 4 \mathrm{~B}$ & 109.2 \\
\hline $\mathrm{N} 1-\mathrm{C} 1-\mathrm{C} 2$ & $111.34(12)$ & $\mathrm{C} 3-\mathrm{C} 4-\mathrm{H} 4 \mathrm{~B}$ & 109.2 \\
\hline $\mathrm{N} 1-\mathrm{C} 1-\mathrm{H} 1 \mathrm{~A}$ & 109.4 & $\mathrm{H} 4 \mathrm{~A}-\mathrm{C} 4-\mathrm{H} 4 \mathrm{~B}$ & 107.9 \\
\hline $\mathrm{C} 2-\mathrm{C} 1-\mathrm{H} 1 \mathrm{~A}$ & 109.4 & $\mathrm{O} 1-\mathrm{C} 5-\mathrm{N} 1$ & $121.49(13)$ \\
\hline $\mathrm{N} 1-\mathrm{C} 1-\mathrm{H} 1 \mathrm{~B}$ & 109.4 & $\mathrm{O} 1-\mathrm{C} 5-\mathrm{C} 6$ & $119.42(12)$ \\
\hline $\mathrm{C} 2-\mathrm{C} 1-\mathrm{H} 1 \mathrm{~B}$ & 109.4 & $\mathrm{~N} 1-\mathrm{C} 5-\mathrm{C} 6$ & $119.09(12)$ \\
\hline $\mathrm{H} 1 \mathrm{~A}-\mathrm{C} 1-\mathrm{H} 1 \mathrm{~B}$ & 108.0 & $\mathrm{C} 5-\mathrm{C} 6-\mathrm{S} 2$ & $111.99(10)$ \\
\hline $\mathrm{C} 1-\mathrm{C} 2-\mathrm{S} 1$ & $111.64(11)$ & $\mathrm{C} 5-\mathrm{C} 6-\mathrm{H} 6 \mathrm{~A}$ & 109.2 \\
\hline $\mathrm{C} 1-\mathrm{C} 2-\mathrm{H} 2 \mathrm{~A}$ & 109.3 & $\mathrm{~S} 2-\mathrm{C} 6-\mathrm{H} 6 \mathrm{~A}$ & 109.2 \\
\hline $\mathrm{S} 1-\mathrm{C} 2-\mathrm{H} 2 \mathrm{~A}$ & 109.3 & $\mathrm{C} 5-\mathrm{C} 6-\mathrm{H} 6 \mathrm{~B}$ & 109.2 \\
\hline $\mathrm{C} 1-\mathrm{C} 2-\mathrm{H} 2 \mathrm{~B}$ & 109.3 & $\mathrm{~S} 2-\mathrm{C} 6-\mathrm{H} 6 \mathrm{~B}$ & 109.2 \\
\hline $\mathrm{S} 1-\mathrm{C} 2-\mathrm{H} 2 \mathrm{~B}$ & 109.3 & $\mathrm{H} 6 \mathrm{~A}-\mathrm{C} 6-\mathrm{H} 6 \mathrm{~B}$ & 107.9 \\
\hline $\mathrm{H} 2 \mathrm{~A}-\mathrm{C} 2-\mathrm{H} 2 \mathrm{~B}$ & 108.0 & $\mathrm{~S} 2-\mathrm{C} 7-\mathrm{H} 7 \mathrm{~A}$ & 109.5 \\
\hline $\mathrm{C} 4-\mathrm{C} 3-\mathrm{S} 1$ & $112.52(10)$ & $\mathrm{S} 2-\mathrm{C} 7-\mathrm{H} 7 \mathrm{~B}$ & 109.5 \\
\hline $\mathrm{C} 4-\mathrm{C} 3-\mathrm{H} 3 \mathrm{~A}$ & 109.1 & $\mathrm{H} 7 \mathrm{~A}-\mathrm{C} 7-\mathrm{H} 7 \mathrm{~B}$ & 109.5 \\
\hline $\mathrm{S} 1-\mathrm{C} 3-\mathrm{H} 3 \mathrm{~A}$ & 109.1 & $\mathrm{~S} 2-\mathrm{C} 7-\mathrm{H} 7 \mathrm{C}$ & 109.5 \\
\hline $\mathrm{C} 4-\mathrm{C} 3-\mathrm{H} 3 \mathrm{~B}$ & 109.1 & $\mathrm{H} 7 \mathrm{~A}-\mathrm{C} 7-\mathrm{H} 7 \mathrm{C}$ & 109.5 \\
\hline $\mathrm{S} 1-\mathrm{C} 3-\mathrm{H} 3 \mathrm{~B}$ & 109.1 & $\mathrm{H} 7 \mathrm{~B}-\mathrm{C} 7-\mathrm{H} 7 \mathrm{C}$ & 109.5 \\
\hline
\end{tabular}




$\begin{array}{llll}\mathrm{C} 5-\mathrm{N} 1-\mathrm{C} 1-\mathrm{C} 2 & -115.63(15) & \mathrm{C} 1-\mathrm{N} 1-\mathrm{C} 5-\mathrm{O} 1 & -175.84(13) \\ \mathrm{C} 4-\mathrm{N} 1-\mathrm{C} 1-\mathrm{C} 2 & 64.06(16) & \mathrm{C} 4-\mathrm{N} 1-\mathrm{C} 5-\mathrm{O} 1 & 4.5(2) \\ \mathrm{N} 1-\mathrm{C} 1-\mathrm{C} 2-\mathrm{S} 1 & -62.22(15) & \mathrm{C} 1-\mathrm{N} 1-\mathrm{C} 5-\mathrm{C} 6 & 3.2(2) \\ \mathrm{C} 3-\mathrm{S} 1-\mathrm{C} 2-\mathrm{C} 1 & 53.57(12) & \mathrm{C} 4-\mathrm{N} 1-\mathrm{C} 5-\mathrm{C} 6 & -176.45(12) \\ \mathrm{C} 2-\mathrm{S} 1-\mathrm{C} 3-\mathrm{C} 4 & -52.50(12) & \mathrm{O} 1-\mathrm{C} 5-\mathrm{C} 6-\mathrm{S} 2 & -86.42(14) \\ \mathrm{C} 5-\mathrm{N} 1-\mathrm{C} 4-\mathrm{C} 3 & 117.12(14) & \mathrm{N} 1-\mathrm{C} 5-\mathrm{C} 6-\mathrm{S} 2 & -73.01(11) \\ \mathrm{C} 1-\mathrm{N} 1-\mathrm{C} 4-\mathrm{C} 3 & -62.59(16) & \mathrm{C} 7-\mathrm{S} 2-\mathrm{C} 6-\mathrm{C} 5 & \\ \mathrm{~S} 1-\mathrm{C} 3-\mathrm{C} 4-\mathrm{N} 1 & 59.54(14) & & \end{array}$

Hydrogen-bond geometry $\left(\AA,{ }^{\circ}\right)$

\begin{tabular}{lllll}
\hline$D-\mathrm{H} \cdots A$ & $D-\mathrm{H}$ & $\mathrm{H} \cdots A$ & $D \cdots A$ & $D-\mathrm{H} \cdots A$ \\
\hline $\mathrm{C} 1-\mathrm{H} 1 B \cdots \mathrm{O} 1^{\mathrm{i}}$ & 0.99 & 2.46 & $3.3490(19)$ & 150 \\
$\mathrm{C} 6-\mathrm{H} 6 B \cdots \mathrm{O} 1^{\mathrm{i}}$ & 0.99 & 2.59 & $3.4427(18)$ & 144 \\
$\mathrm{C} 7-\mathrm{H} 7 B \cdots \mathrm{O} 1^{\mathrm{ii}}$ & 0.98 & 2.45 & $3.3237(19)$ & 148 \\
$\mathrm{C} 3-\mathrm{H} 3 A \cdots \mathrm{S} 2^{\mathrm{iii}}$ & 0.99 & 2.88 & $3.8201(15)$ & 159 \\
\hline
\end{tabular}

Symmetry codes: (i) $x,-y+3 / 2, z+1 / 2$; (ii) $x, y+1, z$; (iii) $x, y-1, z$. 